\title{
Dificultad en el tratamiento del hipertiroidismo por amiodarona. Presentación de un caso
}

\author{
T. PEDRO, S. MARTÍNEZ-HERVÁS, R. LORENTE, M. CATALÁ, J. ORTEGA, J. T. REAL, \\ R. CARMENA
}

Servicio de Endocrinología y Nutrición. Hospital Clínico Universitario. Departamento de Medicina. Universidad de Valencia. Valencia

DIFFICULT TREATMENT OF AMIODARONE-INDUCED THYROTOXICOSIS: A CASE REPORT

\begin{abstract}
RESUMEN
La amiodarona puede alterar la función tiroidea en un 15-20\% de los pacientes que la toman, dando lugar a hipotiroidismo o hipertiroidismo. La sobrecarga de yodo y la toxicidad directa sobre el tiroides inducida por amiodarona pueden provocar hipertiroidismo. Describimos un caso de tirotoxicosis grave por amiodarona que se diagnosticó buscando la causa que descompensó la cardiopatía de base del paciente, condicionando ésta el pronóstico y el manejo terapéutico. El tratamiento precisó un estrecho seguimiento y se basó en dosis elevadas de propiltiouracilo y dexametasona que no controlaron las consecuencias del hipertiroidismo a nivel cardiaco necesitando la tiroidectomia total. Creemos, con la experiencia aportada, que en determinados casos la cirugía es una opción que debería ser planteada con más precocidad.
\end{abstract}

PALABRAS CLAVE: Hipertiroidismo por amiodarona. Antitiroideos de síntesis. Corticoterapia. Tiroidectomia total.

\begin{abstract}
Amiodarone causes changes in thyroid function tests in about 15 $20 \%$ of patients, inducing either hypothyroidism or thyrotoxicosis. The iodine load and the destructive thyroiditis caused by amiodarone produce thyrotoxicosis. We report a case of amiodarone-induced thyrotoxicosis diagnosed when investigating the reason for worsening of cardiac funtion. Prognosis and treatment of cardiac disorder were determined by thyrotoxicosis. The management needed a closed monitoring of thryroid function. Treatment was based on high doses of propylthiouracil and dexametasone, but they couldn't control cardiac condition and surgery was warranted. When amiodarone-induced thyrotoxicosis is refractory to medical treatment, we belive surgery should be considered earlier.
\end{abstract}

KEY WORDS: Amiodarone-induced thyrotoxicosis. Antithyroid drugs. Corticosteroids. Thyroidectomy.

Pedro T, Martínez-Hervás S, Lorent R, Catalá M, Ortega J, Real JT, Carmena R. Dificultad en el tratamiento del hipertiroidismo por amiodarona. Presentación de un caso. An Med Interna (Madrid) 2007; 24: 543-546.

\section{INTRODUCCIÓN}

La amiodarona es un fármaco antiarrítmico de clase III ampliamente utilizado. Es eficaz en el manejo urgente de la fibrilación y taquicardia ventricular sostenida y en el tratamiento crónico de la fibrilación auricular y taquicardias paroxísticas supraventriculares (1).

La dosis diaria habitual de $200 \mathrm{mg}$ proporciona un exceso diario de 7,5 mg de yodo, 50 veces más que la ingesta óptima de yodo diaria recomendada $(0,15-0,20 \mathrm{mg})(2)$. La sobrecarga de yodo, junto a los efectos intrínsecos de la amiodarona, provoca alteraciones en las pruebas de función tiroidea como adaptación fisiológica hasta en un 50\% de los pacientes, con aumentos leves de T4 libre y TSH y descenso de T3 (3). La mayoría permanecen eutiroideos, pero hasta un $15-20 \%$ de pacientes desarrollan hipertiroidismo o hipotiroidismo por amiodarona (2). La incidencia de hipotiroidismo varía entre un 1 y $32 \%$, siendo mayor en regiones con alta ingesta de yodo, en mujeres y en pacientes con enfermedad de Hashimoto subyacente y su tratamiento no suele plantear dificultades (4).

Por el contrario, el hipertiroidismo por amiodarona aparece más frecuentemente en zonas con deficiencia de yodo (10$15 \%$ ), en hombres y en pacientes con bocio multinodular (5). Aunque la mayoría de los casos publicados son leves-moderados, algunos pacientes presentan tirotoxicosis graves, con resistencia al tratamiento con tionamidas por la alta saturación en yodo de la glándula tiroidea. Esta tirotoxicosis agrava la enfermedad cardiaca de base que en general presentan los afectados. En estos casos, tanto el diagnóstico como el tratamiento son difíciles de realizar, como se comenta en nuestro caso clínico.

\section{CASO APORTADO}

Varón de 75 años con antecedentes de exfumador, hipertensión arterial, arritmia completa por fibrilación auricular, cardiopatía isquemia crónica (infarto agudo de miocardio anterolateral en 1998 y

Trabajo aceptado: 18 de junio de 2007 
episodios de angor inestable en 2001, objetivándose una estenosis significativa de 3 vasos coronarios no susceptibles de tratamiento revascularizador mediante coronariografía), insuficiencia cardiaca sistólica (fracción de eyección del 36\% mediante ecocardiografía), portador de un desfibrilador automático implantable (DAI) desde el año 2000 por presentar episodios recurrentes de taquicardias ventriculares sostenidas monomórficas (TVMS), enfermedad pulmonar crónica obstructiva e hiperplasia benigna de próstata. Desde el año 2001, el paciente no había presentado nuevos episodios de angor, ni de crisis de palpitaciones y mantenía un tratamiento diario con amiodarona $200 \mathrm{mg}$. No se objetivaron alteraciones en la función tiroidea durante este período, manteniendo T4 libre $1,2 \mathrm{ng} / \mathrm{dL}(0,8-1,7)$ y TSH 2,27 mcU/ml (0,2-5).

En septiembre del 2004, presentó cuatro episodios de angor inestable en un período de tres meses, acompañados de episodios de TVMS que el DAI no pudo controlar y que revirtieron tras cardioversión eléctrica junto con amiodarona y procainamida endovenosas. Asociaba astenia y debilidad proximal progresiva en los últimos seis meses junto a una pérdida de peso no cuantificada y cambio de carácter con tendencia a la depresión. En la exploración física destacó una frecuencia cardiaca arrítmica de $86 \mathrm{lpm}$, presión arterial de $120 / 70$, temblor fino distal e intensa atrofia muscular de extremidades. No se palpó bocio y no se objetivó proptosis ni alteraciones en la movilidad extraocular. Durante el último ingreso, se solicitó un control de hormonas tiroideas mostrando T4 libre 6,9 ng/dl y TSH 0,01 $\mathrm{mcU} / \mathrm{ml}$. Ante el diagnóstico de hipertiroidismo por amiodarona, se realizó una ecografía tiroidea que mostró una glándula de tamaño normal y morfología conservada con un único nódulo de 8 × $4 \mathrm{~mm}$ de diámetro en lóbulo tiroideo izquierdo. Los anticuerpos antitiroideos fueron negativos [Ac antitiroglobulina $14 \mathrm{U} / \mathrm{ml}(0-115)$, Ac antimicrosomiales 12 U (0-30)] y TSI 0 UI/L $(0-1,5)$. Se retiró la amiodarona y se inició inmediatamente tratamiento con propiltiouracilo $300 \mathrm{mg} /$ día y prednisona $60 \mathrm{mg} / 24 \mathrm{~h}$. A pesar de esta pauta, los valores de T4l aumentaron tras 5 días de tratamiento y el paciente continuó con episodios de angor inestable y descargas repetidas del DAI, con lo que se aumentó el tratamiento a dexametasona $2 \mathrm{mg} / \mathrm{ev} / 6 \mathrm{~h}$ y propiltiouracilo $450 \mathrm{mg} /$ día, objetivando un descenso progresivo de las cifras de T41 (Fig. 1) y progresiva estabilización de la clínica cardiológica. Coincidiendo con el descenso a prednisona $30 \mathrm{mg} / \mathrm{vo} / 12 \mathrm{~h}$ y manteniendo la dosis de propiltiouracilo, se produjo una exacerbación del hipertiroidismo que precisó nuevo aumento de la dosis de corticoides. Como complicaciones durante el ingreso, el paciente presentó una sobredosificación de acenocumarol por el hipertiroidismo precisando plasma fresco congelado y una neumonía nosocomial. Se produjo un empeoramiento de su miopatía proximal atribuida al hipertiroidismo y a la corticoterapia que lo mantuvo encamado y, además, desarrolló una diabetes secundaria al tratamiento corticoideo que fue tratada con insulina.

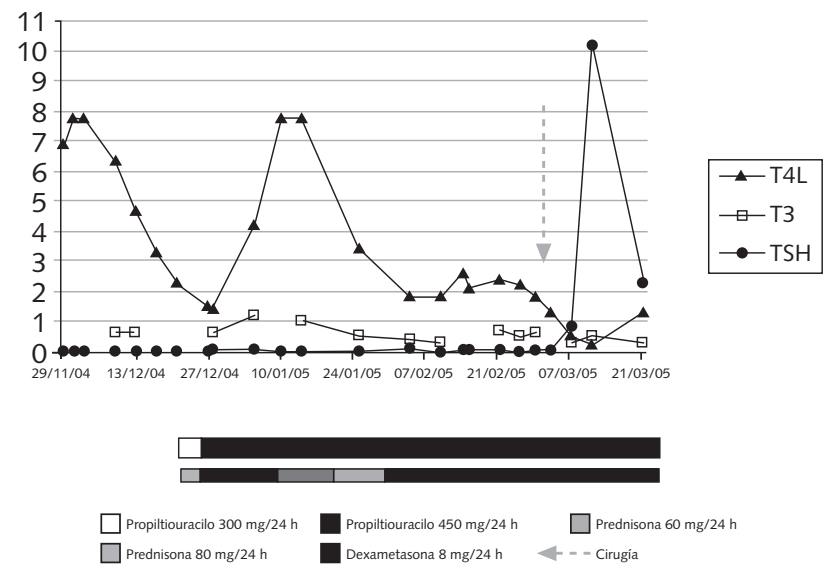

Fig. 1. Niveles de TSH ( $\mathrm{mcU} / \mathrm{ml})(0,27-4,2), \mathrm{T} 4 \mathrm{l}(\mathrm{ng} / \mathrm{dl})(0,93-1,7)$ y T3 $(\mathrm{ng} / \mathrm{ml})(0,8-2)$ durante el tratamiento.
En febrero de 2005, presentó un nuevo episodio de TVMS e insuficiencia cardiaca que precisó nuevamente cardioversión eléctrica y procainamida ev. Ante el fracaso del tratamiento con antitiroideos y corticoides y por las complicaciones asociadas se planteó una opción quirúrgica, realizándose una tiroidectomía total bajo anestesia general.

La anatomía patológica mostró una glándula tiroidea de 6,3 x 4,5 x $2 \mathrm{~cm}$ con aumento difuso de su tamaño e histológicamente se observó un tejido tiroideo con folículos de gran tamaño, distendidos, con aplanamiento del epitelio y contenido coloideo. Estos folículos se encontraban rodeados por zonas de fibrosis e infiltración grasa, con focos de macrófagos espumosos. No se apreció infiltrado linfocitario. Junto a estos folículos se observaron otros, de menor tamaño, con degeneración vacuolada en el citoplasma epitelial (Fig. 2).

Tras la intervención, se inició tratamiento con levotiroxina hasta alcanzar dosis sustitutivas. Actualmente, después de 18 meses de seguimiento, se evidencia una franca recuperación de su estado general, habiendo sufrido un único episodio de TVMS por mal funcionamiento del DAI a los 6 meses de la tiroidectomía.

\section{DISCUSIÓN}

El diagnóstico de hipertiroidismo por amiodarona es complejo debido a las pocas manifestaciones clínicas de tirotoxicosis, predominando las cardiacas. En nuestro caso, sólo objetivamos una pérdida de peso no cuantificada y astenia, al igual que en pacientes de edad avanzada (5). La amiodarona presenta efectos betabloquantes y, por ello, las palpitaciones y taquicardias pueden estar ausentes. Sólo se llegó al diagnóstico con la búsqueda de la causa responsable de las taquicardias ventriculares de repetición. La descompensación de una patología cardiaca subyacente o el desarrollo y/o reaparición de una fibrilación auricular en un paciente tratado con amiodarona debe hacernos sospechar esta patología (7).

La anticoagulación es una terapia frecuente en estos pacientes y debe ser monitorizada estrechamente. La amiodarona inhibe enzimas del citocromo $\mathrm{P} 450$ y produce una interacción farmacocinética con el metabolismo hepático de los anticoagulantes; provoca una disminución del aclaramiento plasmático y potencia el efecto anticoagulante. Por otro lado, la tirotoxicosis también potencia el efecto de los anticoagulantes, pero mediante interacción farmacodinámica al disminuir algunos factores de coagulación (II, VII) (8). En nuestro caso la situación de hipertiroidismo grave, a pesar de haber retirado la amiodarona, produjo una sobredosificación de acenocumarol con INR indetectable que precisó tratamiento con plasma fresco congelado.

Las características histopatológicas de este caso coinciden con la serie de hipertiroidismos por amiodarona descrita por Smyrk y cols. (9). La característica diferencial más importante es la involución de los folículos tiroideos distendidos por coloide así como la asociación con cambios degenerativos y de destrucción folicular y la presencia de fibrosis con un inconstante infiltrado de células inflamatorias junto a esta. En nuestro caso no aparece el infiltrado inflamatorio que atribuimos al tratamiento prolongado con corticoides.

Se distinguen dos tipos de hipertiroidismo por amiodarona según su patogenia (10). El tipo 1 está inducido por yodo y se desarrolla en pacientes con enfermedad tiroidea de base (bocio multinodular o enfermedad de Graves latente) por aumento de síntesis y liberación de hormona tiroidea. El tipo 2 es una tiroiditis destructiva con liberación de hormona preformada debida a la toxicidad directa del fármaco, sin enfer- 

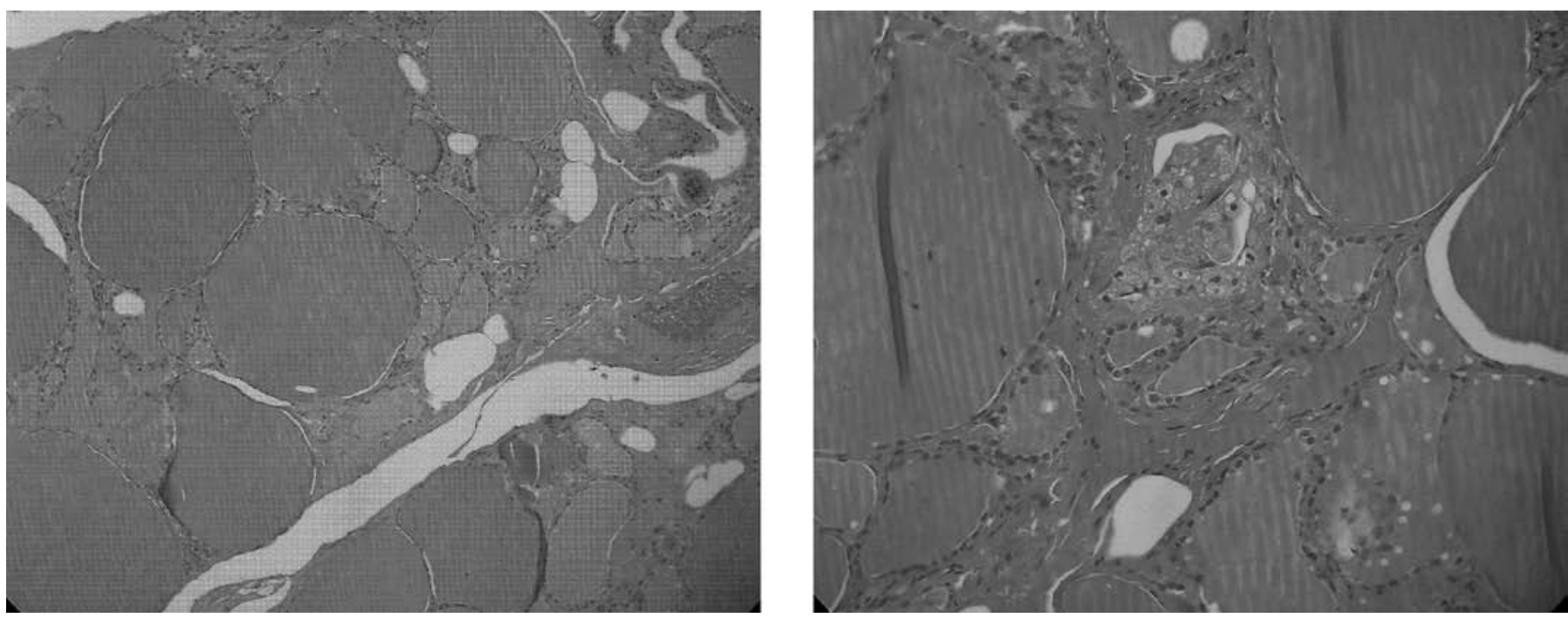

Fig. 2. Imagen microscópica de la glándula tiroidea (HE x4/×20) Se aprecia distensión de los folículos por material coloide junto a zonas de fibrosis y degeneración grasa y vacuolada.

medad tiroidea previa. En la práctica, se describen también formas mixtas con características de ambos tipos (7). La importancia teórica de diferenciarlos reside en optimizar el tratamiento: antitiroideos de síntesis en el tipo 1 para inhibir la producción hormonal y corticoides en el tipo 2 por sus efectos antiinflamatorios (2).

Existen hallazgos exploratorios y pruebas complementarias que nos ayudan a diferenciarlos, si bien no son concluyentes, tales como: el antecedente de patología tiroidea y la palpación de bocio multinodular, la gammagrafía con 131 I (captación disminuida en ambos por bloqueo de captación del trazador por los altos niveles de yodo circulantes en el tipo 1 y por la destrucción tiroidea en el tipo 2 (6); aunque también se ha descrito un aumento de captación del trazador en tipo 1 (2) y la ecografía doppler tiroidea (actualmente la es la prueba con mayor sensibilidad y especificidad que muestra una elevación del flujo sanguíneo tiroideo en el tipo 1 y por el contrario una disminución en el tipo 2)(11).

En nuestro caso, no se solicitó una gammagrafía ya que no modificaba la actitud terapéutica y la ecografía-doppler tiroidea estaba dificultada por la falta de experiencia en esta técnica. Sin embargo, la ausencia de antecedentes de patología tiroidea y la exacerbación del cuadro coincidiendo con los descensos de corticoides nos sugieren que predominaba un componente de tiroiditis.

Se inició el tratamiento con la asociación de antitiroideos y corticoides dada la gravedad clínica del caso que requería un abordaje terapéutico inmediato. También, en la práctica clínica, se recomienda iniciar con ambos tipos de fármacos en las situaciones en que no se puede diferenciar entre un tipo $u$ otro de hipertiroidismo (2). Los antitiroideos propuestos son el metimazol a dosis de 40-80 mg/día o propiltiouracilo 400-800 $\mathrm{mg}$ /día, ya que los depósitos intratiroideos de yodo dificultan sus efectos inhibitorios sobre la síntesis tiroidea (2). En nuestro caso, optamos por el propiltiouracilo. Pensamos que, debido a sus efectos sobre la disminución de la conversión periférica de T4 en T3, podía aportar más beneficio que el carbimazol una vez retirada la amiodarona.

En cuanto a los corticoides, se recomienda la prednisona (40-60 mg/día) disminuyendo gradulamente las dosis con la normalización de T41 $(6,7,9)$. También está indicada la dexametasona a dosis de $2 \mathrm{mg} / 6 \mathrm{~h}$ al inhibir la secreción hormonal y la conversión periférica de T4 a T3 junto a sus efectos inmunosupresores (12), objetivando una rápida mejoría de la función tiroidea en una semana en la mayoría de los casos leves (3). Existen exacerbaciones del hipertiroidismo con descensos rápidos en la dosis de corticoides que responden al aumento de dosis, por lo que se recomiendan tratamientos prolongados (1-2 meses) y retiradas progresivas (13), tal y como realizamos en el caso presentado. Así mismo, las recurrencias del hipertiroidismo son comunes, debiendo mantener un estrecho seguimiento del paciente. En nuestro caso, la disminución de la dosis de corticoides hasta las dosis recomendadas en un periodo de 30 días, provocó una nueva exacerbación del hipertiroidismo, precisándose altas dosis de corticoides para mantener el control hormonal. Objetivamos una buena respuesta con dexametasona, que podría estar en relación a su mayor efecto antiinflamatorio. La escasa retención hídrica, por la ausencia de efecto mineralcorticoide de la dexametasona, permitió un mejor manejo del balance hídrico en la situación de insuficiencia cardiaca de nuestro paciente.

La retirada de amiodarona esta recomendada cuando sea posible junto con antitiroideos a dosis altas $(3,13)$. La amiodarona inhibe la conversión de T41 a T3 y tiene efectos betabloqueantes, por lo que deberemos valorar su retirada, si la situación cardiaca lo permite, y sustituirla por otro antiarrítmico por el riesgo de recurrencia de la arritmia (6). Además, tiene una vida media larga (aproximadamente 100 días) debido a su gran lipofilia y el depósito en tejido graso, lo que no permitirá objetivar una respuesta inmediata con su supresión. El hipertiroidismo puede aparecer desde meses a varios años desde el inicio del tratamiento e incluso tras su retirada (2) y no se relaciona con la dosis de amiodarona acumulada (5).

Cuando la amiodarona no se ha podido retirar o falla el tratamiento médico, como ocurrió en el caso presentado, la tiroidectomía representa una alternativa eficaz $(2,14)$. La intervención quirúrgica en pacientes no controlados supone un elevado riesgo de arritmias y crisis tirotóxica, por lo que se valorará cada situación individualmente. El ácido iopanoico ha demostrado utilidad en controlar el hipertiroidismo previo 
a la cirugía (15). En nuestro caso se optó por una tiroidectomía total al no alcanzar un buen control hormonal a pesar de las altas dosis de tionamidas y corticoides, la persistencia de inestabilidad cardiológico y la necesidad de iniciar el descenso de dosis de corticoides por sus efectos secundarios.

En resumen, nuestro paciente muestra el complejo manejo terapéutico en determinados casos de hipertiroidismo por amiodarona en los que la coexistencia de patología cardiaca grave de base condiciona la actitud y el tratamiento individua-

\section{Bibliografía}

1. Reiffel JA, Estes II NA, Waldo AL, Prystowsky EN, Di Bianco R. A consensus report on antiarrhythmic drug use. Clin Cardiol 1994; 17 : 103-16.

2. Martino E, Bartalena L, Bogazzi F, Braverman LE. The effects of amiodarone on the thyroid. Endocr Rev 2001; 22: 240-54.

3. Newman CM, Price A, Davies DW, Gray TA, Weetman AP. Amiodarone and the thyroid: A practical guide to the management of thyroid dysfunction induced by amiodarone therapy. Heart 1998; 79: 121-7.

4. Martino E, Aghini-Lombardi F, Mariotti S, et al. Amiodarone iodineinduced hypothyroidism: Risk factors and follow-up in 28 cases. Clin Endocrinol (Oxf.) 1987; 26: 227-37.

5. Trip MD, Wiersinga W, Plomp TA. Incidence, predictability and pathogenesis of amiodarone-induced thyrotoxicosis and hypothyroidism. Am J Med 1991; 91: 507-11.

6. Basaria S, Cooper D. Amiodarone and the thyroid. Am J Med 2005; 118: 706-14.

7. Rajeswaran C, Shelton RJ, Gilbey SG. Management of amiodaroneinduced thyrotoxicosis. Swiss Med Wkly 2003; 133: 579-85.

8. Kurnik D, Loebstein R, farfel Z, Ezra D, Halkin H, Olchovsky D. Complex drug-drug-disease interactions between amiodarone, warfarin and the thyroid gland 2004; 83: 107-13. lizado de cada caso. Destacamos la utilidad del propiltiouracilo con el que se mantuvieron cifras de T3 en el límite bajo desde el inicio del tratamiento y la necesidad del descenso lento de las altas dosis de corticoides que podría haber evitado la exacerbación del hipertiroidismo. La resistencia al tratamiento medico a pesar de dosis altas de fármacos requiere un estrecho seguimiento del paciente y, posiblemente, la necesidad de plantear una opción quirúrgica más precoz en grupos de alto riesgo como el caso que presentamos.

9. Smyrk TC, Goellner JR, Brennan MD, Carney JA. Pathology of the thy roid in amiodarone-associated thyrotoxicosis. Am J Surg Pathol 1987; 11: 197-204.

10. Bartalena L, Grasso L, Brogioni S, et al. Serum interleukin-6 in amiodarone-induced thyrotoxicosis. J Clin Endocrinol Metab 1993; 78: 423-7.

11. Eaton SE, Euinton HA, Newman CM, et al. Clinical experience of amiodarone-induced thyrotoxicosis over a 3-year period: Role of colour-flow Doppler sonography. Clin Endocrinol (Oxf.) 2002; 56: 33-8.

12. Davies TF, Larsen R. Thyrotoxicosis. In: Larsen PR, Kronnenberg HM, Melmed S, Polonsky KS, editors. Williams textbook of endocrinology. $10^{\text {th }}$ ed. Philadelphia: Elsevier Science; 2003, p. 374-421.

13. Bartalena L, Brogioni S, Grasso L, Bogazzi F, Burelli A, Martino E. Treatment of amiodarone-induced thyrotoxicosis, a difficult challenge: Results from a prospective study. J Clin Endocrinol Metab 1996; 81: 2930-3.

14. Houghton SG, Farley DR, Brennan MD, van Heerden JA, Thompson GB, Grant CS. Surgical management of amiodarone-associated thyrotoxicosis: Mayo Clinic experience. World J Surg 2004; 28: 1083-7.

15. Bogazzi F, Miccoli P, Berti P, et al. Preparation with iopanoic acic rapidly controls thyrotoxicosis in patients with amidarone-induced thyrotoxicosis before thyroidectomy. Surgery 2002; 132: 1114-7. 